\title{
My First Step - A Paradigm Shift to Ending Violence against FSWS (Female Sex Workers): A Pakistani Study
}

\section{Benish Shabbir* and Hassan Siddique}

Capital University of Science and Technology, Management and social sciences, Islamabad Expressway, Kahuta Road, Zone-V Islamabad, Islamabad, Islamabad Capital Territory 44000, Pakistan

\begin{abstract}
In this study we investigate and explore the violence against FSWS. There are a lot of laws and acts passed which protected the rights of women such as Convention on the Elimination of All Forms of Discrimination against Women (CEDAW) 1996, Prevention and Control of Human Trafficking Ordinance (2002), Prevention and Control of Human Trafficking Rules (2004) and Protection of Women (Criminal laws Amendment) Act (2006). All four of the documents mentioned above, have many gaps and lack of conceptual clarities. For instance, CEDAW is very general in nature and serves more as a guiding principle, interpreted solely by the discretion of the judges. This study is not only discussing the rights of FSWS but tell how to implement the laws and act on them so that they feel protected as a woman.
\end{abstract}

Keywords: Ending violence; Rights of women; Law; Policy

\section{Law(s), Policy(s) or Action Plan(s)}

In addition, the State's unwillingness to ratify the Optional Protocol to CEDAW, which compliments CEDAW and establishes effective mechanisms for enforcement of the rights specified therein, is another grey area of CEDAW's implementation in Pakistan. Similarly, laws on trafficking are full of gaps like conceptual clarity on different categories of trafficking, cases of trafficking in the guise of marriage, issue of trafficking within Pakistan's borders, low punishments, and no focus on rehabilitation of victims [1-5].

The amended Hudood Ordinances, that were responsible for sending many women to prison on charges of sexual relationships outside marriage, have done commendable job by decreasing the cases of custodial violence. Nevertheless, the State continues to avoid the passage of amendments (e.g., domestic violence, and social protection) that are considered more controversial due to the fact that they might affect the traditional set up of the family and society $[6,7]$.

Though, we cannot establish FSWs as direct beneficiaries of these documents, however, we expect to change the patriarchal behaviors by involving the FSWs as formally structured Community Based Organizations, with capacities to voice their problems and suggest solutions in keeping with Rights Based Approach. Being the most marginalized group amongst women, their involvement as Actors could contribute to the larger goal of sustainable progress towards women empowerment, altogether.

Published evidence on previous initiatives shows that most of the studies predominantly address the issues related to prevention of HIV/ AIDS and SRH occasionally referring to the social dimensions of the plight of FSWs and their families. Accordingly, the needs identified by various assessments and evaluations, we propose to work in partnership with FSWs and their families to EVAW for their socioeconomic empowerment and to create enabling environment where they could exercise basic human rights. We intend to mobilize FSWs to establish self-help groups, and to train them in developing linkages with national and international forums to make their voices heard.

\section{Problem Analysis}

Commercial sex is illegal in Pakistan, thus leaving those involved in the trade exposed to all sorts of stigma, denial, discrimination, violence, and abuse. The interplay of personal gender biases affect the implementation of women rights and protection related legislations in a way that does not induce any improvement on the incidents of violence against FSWs which is perpetrated from relatives, clients, and general public.

According to "New Face of Female Sex Work in Pakistan: Need for Innovative Interventions", (Narjis and Quresh 2011).The most common perpetrator of physical violence against FSWs is husbands (66\%), followed by the clients (38\%), and neighbors $(24 \%)$. On the other hand, in case of sexual abuse police tops the list (43\%), followed by husbands (34\%), and other favorite sexual partner (33\%). Therefore, we conclude that attitudinal and behavior changes of key stakeholders are vital to ending VAW in general and FSWs in particular. Because the Illegality that surrounds their profession pushes them on such dubious grounds that deprive them of attaining social justice.

Therefore, we proposed to furnish FSWs with a platform where they could possibly familiarize the world with their miseries; and to establish a forum where they can make their voices heard and understood. Regardless of why people do sex work, they have the right to self-determination, should be treated with dignity and deserve equal protection under the law.

According to the UNAIDS Guidance Note on HIV and Sex Work, p. 7, the UN system "affirms the universality, inalienability and interdependence of rights, and promotes and supports their application in practice, including for sex workers, their clients and otherwise in the context of sex work, even where sex work is criminalized."

*Corresponding author: Shabbir B, Student, Capital University of Science and Technology, Management and social sciences, Islamabad Expressway, Kahuta Road, Zone-V Islamabad, Islamabad, Islamabad Capital Territory 44000, Pakistan, Tel: +923419482511/+923348334939; E-mail: ben.punctual@gmail.com

Received March 01, 2017; Accepted March 18, 2017; Published March 25, 2017

Citation: Shabbir B, Siddique H (2017) My First Step - A Paradigm Shift to Ending Violence against FSWS (Female Sex Workers): A Pakistani Study. Intel Prop Rights. 5: 184. doi: 10.4172/2375-4516.1000184

Copyright: $\odot 2017$ Shabbir B, et al. This is an open-access article distributed under the terms of the Creative Commons Attribution License, which permits unrestricted use, distribution, and reproduction in any medium, provided the original author and source are credited. 


\section{Which social actors does the study seek to engage with and influence}

Fundamentally, we aim to respond to the needs of the community by encouraging community mobilization and critical consciousnessraising among FSWs. For this purpose we have divided the social actors into three broad categories:

The female sex workers: This category includes the females who are the providers of sexual and/or other bodily services to the clients. This group is the potential victim of violence and abuse.

The gatekeepers/co-workers: This category includes the spouses/ relatives, other co-workers (pimps, the madams, aunties), rickshaw drivers, shopkeepers, etc. This category mostly involves those who deal with the client, receive payments, decide meeting place, etc.

Others: This category involves law enforcement bodies, CSOs, clients, media, religious leaders, general public, health service providers, and educational institutions. We intend to involve, sensitize, build capacity of FSWs, so that they could understand and protect their rights. Having involved this group, we would ensure the sustainability of this project, as they can manage, run, and sustain the CBOs, even after we would retreat.

The gatekeepers are important because of their status in the power relations running the sex industry. They are the connecting links between the FSWs and their clients. Therefore, it is important to sensitize them and build their capacity to protect the rights of the FSWs and to advocate and convince the clients about basic human rights and protection issues of FSWs.

The third group mostly comes from middle to high income backgrounds and has deep roots in the society; and play important role at the higher level of power relations, i.e., institutional and government level, the policy makers, public opinion makers, the private sector, etc. Having involved this group, we anticipate a culturally sensitive cognitive-behavior change to introduce long term structural changes that would contribute to ending violence against women.

\section{Why does the study want to engage with and influence the stated social actors?}

The dilemma of FSWs is exacerbated by the socio-political and legal challenges that are a part and parcel of our society which is driven by religious, ethnic, social, and gender-based denominators. In order to address the societal norms, we propose to engage FSWs to empower themselves to exercise their basic human rights as citizens of Pakistan. The influence that we anticipate is to improve their living conditions, de-stigmatize their socio-economic status, and to provide them with a well-coordinated platform whereby they could share experiences, discuss issues, and find solutions.

As gatekeepers play a vital role in linking FSWs and human rights defenders (Law enforcement agencies, CSOs, and media), therefore, we anticipate a supportive behavioral change which would result in increased reporting of the incidents of violence against the FSWs. In this regard, we also expect a more sensitized de-stigmatized recording of the First Investigation Report (FIR) against the offenders in all cases of violence against FSWs irrespective of their profession, ethnic background, language, etc. [8].

From media, policy makers, religious leaders, and CSOs, we expect sustainable contribution to introduce an enabling environment where the power relations, which disfigure the shape of the world of FSWs and their families, could be transformed.

These changes are important to achieve because we have entered in an era of Sustainable Development Goals, where it is inevitable that we introduce action plans that focus on sustainability and are community led so that the stories of achievements in ending VAW go beyond project-end reports.

\section{Contribution of the study}

The overall vision of the study is to empower FSWs that will help in reducing the stigma and discrimination and also violence against women by the society and service providers. It will be done through participatory approach by identifying community leaders and sensitization of the FSWs about their rights and self-esteem; this will lead to formation of support groups and CBOs. These groups will be provided support to build their capacity in human rights and advocacy so that FSWs as a group are more equipped to raise their voice and to protect their rights.

In addition, as a cross cutting theme, the study aims to bring empowerment with prevention of HIV/AIDS through progressive capacity building of FSWs on the rights to bodily integrity, and the highest attainable standard of physical and mental health (including access to sexual and reproductive health care services). As an outcome of our study strategies, we anticipate a reduction in the occurrence of incidents of violence against FSWs and an increase in sensitized reporting (both by media as well as law enforcement agencies) of the same incidents. By implementing our step-by-step approach we anticipate to develop the capacities of the community to face the challenge of stigma and discrimination, and to evolve as an empowered group by addressing the structural causes that lead to the creation of marginalized communities, hence contributing to the social, economic, and moral degradation of the overall society.

\section{Major Outcomes of the Study}

- To sensitize FSWs about their rights and self-esteem.

- To establish formal, structured, and community based self-help groups, to build their cohesive voice to end violence against FSWs, increased participation in their own development, and to advocate for the protection of their rights.

- To empower FSWs through increased awareness of their SR\&HR; and to achieve significant decrease in the spread of HIV/AID and STIs.

- To enhance organizational, technical and project management capacity of CBOs established under the study.

- To ensure their representation at national, regional, and international networks and forums.

- To create an enabling environment through sensitization and awareness sessions of key stakeholders especially law enforcement agencies, community gatekeepers, media, religious leaders, and policy and decision makers to reduce incidents of human rights abuses of FSWs.

- Challenge stigma and discrimination against sex workers, their families and partners, co-workers, and others involved in sex work.

- To go beyond raid, rescue, and rehabilitation; and create enabling environment through behavior change amongst law enforcement agencies to involve sex worker rights based organizations against trafficking, since sex worker-led organizations have the trust of the 
community and thus are often able to identify children and adults that are victims of trafficking.

We believe that this approach of nipping the causes of social injustices would result in many spin-off effects like decreasing the spread of HIV/AIDS; ending VAW, fall in the acts of crime against women, etc., by bringing an overall social balance.

\section{Piloting a Promising Approach}

Our proposed project has been inspired from the proven Indian models of Durbar Mahila Samanwaya Committee (DMSC), a brothelbased sex work project in Sonagachi, and the Ashodaya Samithi project, a CBO for street-based sex workers based in Mysore. These initiatives have become widely diffused models labeled as "empowerment approach" which prioritize community mobilization and structural interventions. Our desk review has shown that these kinds of interventions for ending violence against women have not yet been implemented in Pakistan, though we have come across suggestions on changing the approach on prevention of HIV/AIDS and STIs.

Majority of the studies with FSWs in Pakistan focus on service delivery packages and included: recruitment or encouragement of FSWs to visit a clinic for STI screening; guiding FSWs to the clinic; diagnosis and treatment; education in HIV and STI prevention and in condom negotiation skills; condom demonstration, promotion and distribution; general health education; referrals for clinical services; risk-reduction education or counseling; distribution of IEC materials and safer-sex kits; and awareness raising of available clinical services. Having reviewed the FSW based studies, we come to know that very few appear to have focused on community-led service provision or were concerned with building the capacity of the FSW community to change their circumstances. Indeed, the model that we intend to replicate in proposed provinces in Pakistan need several years to bring about significant social change, as projects like Ashodaya and DMSC took over a decade to develop into complex community empowerment processes.

However, time is not the only variable that helped in achieving the desired results, favorable environment to introduce formal structures of CBOs is equally important factor. Empowering sex workers in India to reduce vulnerability to HIV and sexually transmitted diseases the
Ashodaya and Sonagachi projects gradually evolved into strategies incorporating empowerment. These models were initially simple interventions focusing on the prevention of HIV/AIDS and STIs amongst the sex workers. However, with the passage of time these interventions turned into more complex models with well-articulated and formal structures of CBOs.

\section{Conclusion}

This study introduced community based monitoring mechanism to ensure transparency, accountability, and community ownership. There is a need to give FSWs better health education so that they can judge their way of earning either it is good or bad for them. Information about HIV and sexual health is also a phenomenon that is important for them to understand. Knowledge and community mobilizing efforts by this social capital created and that is used for the welfare and positive influence so that they motivated to other earnings rather that sex. There must be such type of CBOs that societal, lawful and psychological support for female who are involved in such type of sex work. There should be need to provide appropriate medical services so that they come back to their life.

\section{References}

1. UN Women (1996) Convention on the Elimination of All Forms of Discrimination against Women (CEDAW), USA.

2. An Ordinance to prevent and control human trafficking (2002) Prevention and Control of Human Trafficking Ordinance.

3. Lix (2004) Prevention and Control of Human Trafficking Rules.

4. Islamic S (2006) Protection of Women (Criminal laws Amendment) Act. Islamic Studies, Islamic Research Institute, International Islamic University, Islamabad.

5. Lau M (2007) Twenty-Five Years of Hudood Ordinances. A Review Wash \& Lee L Rev 64: 1291.

6. Narjis R, Fozia Q (2011) A report on "New Face of Female Sex Work in Pakistan: Need for Innovative Interventions. Pak J Public Health 1: 28-35.

7. Uniting the world against AIDS (2012) A report on UNAIDS Guidance Note on HIV and Sex Work. Unaids 20 Avenue Appia, Ch-1211 Geneva 27, and Switzerland.

8. Swendeman D, Basu I, Das S, Jana S, Rotheram-Borus MJ (2009) Empowering sex workers in India to reduce vulnerability to HIV and sexually transmitted diseases. Social science \& medicine 69: 1157-1166. 